\title{
Enhancement Of Features In Galaxy Images
}

\section{S. Djorgovski}

S. Djorgovski, "Enhancement Of Features In Galaxy Images," Proc. SPIE 0627, Instrumentation in Astronomy VI, (13 October 1986); doi: $10.1117 / 12.968146$

SPIE. Event: 1986 Astronomy Conferences, 1986, Tucson, United States 


\title{
ENHANCEMENT OF FEATURES IN GALAXY IMAGES
}

\author{
S. Djorgovski
}

Harvard-Smithsonian Center for Astrophysics, 60 Garden St., Cambridge, MA 02138, USA.

\begin{abstract}
I describe and compare several image enhancement techniques, useful for morphological analysis of galaxian (or cometary) images. Such techniques can be used to search for, and investigate the properties of dust lanes, stellar disks or rings, jets, shells, tidal distortions, etc. Applications of the techniques are illustrated on CCD images of the peculiar galaxy Arp 230; this object has a rich morphology, indicative of a merger of two disk galaxies.
\end{abstract}

\section{Introduction}

Visual irnages are, and may for ever be, the principal means of conveying astronomical information, concepts, or ideas. This is true independent of the wavelength in which the displayed information was obtained. Most of the modern astronomical data and many theoretical simulations are produced in a digital image form. It is only proper to try to extract most from such images, and image-processing computer time is generally more affordable or available than the telescope or a supercomputer time. In this paper I compare several digital image enhancement techniques, none of which are new, but some of which are not yet widely used by the astronomers. I address here image enhancement rather than image restoration techniques: the former modify an image in a way which makes some of its features appear more prominent; the later (such as the seeing deconvolutions, etc.) try to compensate for some known distortion, or presence of a known form of noise.

The two main ideas which I would like to convey are: (1) the choice of an optimal technique in a given situation depends strongly on the particular details and desiderata of the problem, but, (2) techniques which allow the astronomer to use some a priori information, which is not always and/or entirely contained in the image itself, are likely to produce better results. Perhaps the best illustration of the second principle is the maximum entropy method (MEM; cf. the reviews by Wells ${ }^{1,2}$ ), whose advantages and successes are mainly due to the explicite use of the known functional forms of noise, the PSF, and the physical requirement that there are no negative photons. Since the MEM received adequate attention (and even some undue propaganda?) in the literature, I will not address it further in this paper.

One standard situation, on which I will draw my examples, is enhancement of features which "do not quite belong" in galaxy images. As a fruitful target, I will use images of the peculiar galaxy Arp 230 (= IC 53?). This galaxy contains a warped stellar ring or disk, a dust lane, a set of shells $\mathbf{s}^{3,4}$, and other possible morphological signatures of a tidal encounter ${ }^{5}$. The data set consists of images obtained with the CTIO $1.5-\mathrm{m}$ telescope and with an RCA $512 \times 320 \mathrm{CCD}$, on the night of 21 June 1985 UT. Two B images with 3 and 5 minute exposures, and one each of V, R, and I, 8 minute exposures were obtained. The pixel scale was 0.542 arcsec, and seeing (PSF FWHM) about 1.5 arcsec. The images were flattened, cleaned of cosmic rays, sky subtracted, and shifted in positional coincidence by using a fractional pixel rebinning. A stack of all images was used for all examples shown here. Figures 1 and 2 show this galaxy as it appears at different contrast levels. Only the central $160 \times 160$ pixels, which cover most of the galaxy, are shown in the Figures. All fields are 86.7 arcsec squares, with North on the top, East to the left.

\section{Discussion of Particular Techniques}

We are faced with a situation where low-contrast features (e.g., the shells) are embedded in a high-contrast background, viz., the global light distribution of a galaxy. Thus, the problem can be approached as a background-removal, or as a high-pass filtering. Another way to look at it is as a "misdistribution of the dynamical range": low-contrast features of interest are lost in the steep, centrally concentrated background, which dominates the image. The high-pass filtering aspect signals another difficulty: enhancement of small-scale features will as a rule enhance the noise as well, and some smoothing may be necessary in the final stages of processing.

Fourier filtering is one of the standard tools which may be used here. Figure 3 shows the results of a high-pass filtering: the processing was done on a $256 \times 256$ image, central portion of which is shown here. The shape of the filter (in the amplitude space) was an inverted cone with a Gaussian falloff. This kind of filter effectively removes the background, leaving the prominent nuclear stellar disk and the dust lane. However, the inescapable ringing near high-contrast features makes the interpretation of light and dark somewhat ambiguous.

Another simple high-pass (or edge) enhancement is application of a two-dimensional derivative (Laplacean) operator, which can be accomplished numerically as follows: First, form the differences

$$
\Delta_{1}=I(i, j)-I(i-1, j), \quad \Delta_{2}=I(i, j)-I(i+1, j), \quad \Delta_{3}=I(i, j)-I(i, j-1), \quad \Delta_{4}=I(i, j)-I(i, j+1),
$$

where $I(i, j)$ represents the pixel value at the $j$-th row and $i$-th column, etc. The output pixel value is simply

$$
O(i, j)=\left(\Delta_{1}^{2}+\Delta_{2}^{2}+\Delta_{3}^{2}+\Delta_{4}^{2}\right)^{1 / 2}
$$


An example of this operation is illustrated in Figure 4: the nuclear warped disk and dust lane system shows well, but there is also a trace of the shells, which were not apparent in the Fourier-filtered image. Note, however that the intensity maxima become zeroes (ridges become canyons, peaks become craters), as the derivatives $\Delta_{k}$ reach zero values there. This method seems particularly useful in enhancement of small features, e.g., intricate filamentary patterns often seen in Galactic nebulae.

Similar to this technique is the directional (one-dimensional) gradient enhancement, illustrated in Figures 5 and 6 . The operation is simply to shift the image in a well-chosen direction by a small number of pixels, and subtract it from, or divide it by the original image. It helps very much to presmooth the image which is being used for the division or subtraction: it is well known that differentiation of noise produces even more noise... The two versions of this technique, subtraction (Fig. 5) and division (Fig. 6) differ substantially in their effect: the subtraction gradient preserves intensity contrasts, and the low-level features are lost; the division "gradient" removes the background, and enhances features at all intensity levels, but for a price of an enhanced noise. A version of this technique ${ }^{6}$ can be used to remove the stellar background trails in tracked cometary images.

When dividing by a noisy image, one must excercise some caution, as the properties of "division noise" may be rather unpleasant. Some softening can help: a method I like to use is to find the r.m.s. of the noise (from the image histogram, say), and truncate the denominator image at a 1- $\sigma$ level; I then add a constant $(\sim 1 \sigma)$ to the image. Such "softened division" (so familiar in N-body simulations) produces much better noise behavior. This procedure is somewhat similar to the Wiener filtering, but in the real domain, and may even be defensible on statistical grounds.

The morphological features of our test object, Arp 230, are very prominent, but the two gradient techniques described above do not perform that well in most astronomical applications. The reason is that most galaxy (or comet) images are dominated by a strong radial intensity gradient, which gets enhanced as well, and often drowns the finer, embedded details. The solution is to use a polar coordinates equivalent of the gradient technique, with the pole set at the intensity peak. Operationally, the image is rotated by a small angle around this center, and/or radially stretched (squeazed) by a factor close to unity; this "shifted" image can then be used for division or subtraction in a way analogous to the directional gradient enhancement described above. Some examples are shown in Figures 7 - 9. Rotation alone (Fig. 7) will effectivelly enhance all deviations from an azimuthal symmetry, such as dust lanes, stellar disks or bars, or jets. Radial stretch alone (Fig. 8) will enhance all radial intensity jumps, which may otherwise follow the isophotal shapes, such as shells, face-on rings, etc. A combination of radial stretch and rotation (Fig. 9) will do both. The distortion and noise increase in proportion to the radius, and one must chose the shift and stretch factors carefully, by assesing first where are the possible features of interest. This technique may prove very efficient in a systematic search for shells around elliptical galaxies. It was also used with a substantial gain by Sekanina and Larson on digitized plates of 1910 apparition of $\mathrm{P} / \mathrm{Halley}^{7,8}$.

Thus, by using a known property of an image, one can do better than by applying a general technique (such as the Fourier filtering), which is oblivious to that kind of information. This principle can be carried further, and to a great advantage. We are, in effect, dealing with the problem of background removal; what is needed then is a realistic, relatively noise-free model of the background, against which one can explore the possible morphological features.

One simple way of producing such smooth background is from the image itself. For this purpose I find it useful to combine different smoothing techniques. A first step may be squeazing of the image by an integer factor in each direction; this increases the signal-to-noise, reduces the size of small-scale features and stars to a few pixels, and makes the image sizes more manageable. The next step is median filtering: it disposes of any glitches, stars, and other sharp features. Notice that the process is nonlinear and non-flux-preserving, those being valuable, but tricky properties of nonparametric (median) filtering. The resulting image is then smoothed and expanded to the original size; I like to interleave successive expansions (by a factor of two) and boxcar smoothings. Bilinear, or spline interpolation can be used to reexpand the image to a larger size, and it is a smoothing procedure in itself. The end product is a very smooth image, which maintains most of the global shape of the original, but excludes the fine structure. The original image can then be divided by this smoothed version. An example is shown in Figure 10: both the central structure and the outter shells are evident. Division of an image by its smoothed version almost always causes some ringing, by analogy with the Fourier filtering; this tendency is suppressed somewhat by the median filtering.

A more powerful, but not always justifiable method is to construct a pure elliptical-isophote model of the galaxy, with major axis intensity, ellipticity and position angle (PA) varying as in the real galaxy. In order to do this, detailed surface photometry is necessary (see refs. 9 and 10 for more details). Galaxian isophotes are not always elliptical, but this is a fairly good approximation; introduction of higher-order azimuthal Fourier terms may help, but it would complicate the modeling procedure substantially. This technique has been used with great success in searches for dust lanes and stellar disks in early-type galaxies ${ }^{10,11,12,13}$. It is illustrated here in Figures $11-13$.

A word of warning is due here: it may be misleading to assume some form of intensity (or ellipticity, or PA) profiles for galaxies which are investigated in this way. These profiles should be measured, and may be smoothed. Subtracting an inapropriate profile (e.g., a de Vaucouleurs's $r^{1 / 4}$ law) causes artifacts which can be grossly overinterpreted, as it was occassionally done in the literature. 


\section{Acknowledgements}

This paper was completed during my stay at the Astronomy Department, University of California at Berkeley, whose hospitality and stimulative atmosphere I gratefully acknowledge. The data on Arp 230 were obtained in collaboration with Ivan King, whose NASA contract NAS5-28086 financed the computing. The data were obtained at the Cero Tololo Interamerican Observatory, which is operated by Aura, Inc., under a contract with the NSF. I thank Patricio Ugarte for assistance at the telescope, and Carl Vuosalo and Abe Oren for help with CCD data processing. The elliptical galaxy modeling routine was written by Tod Lauer. I acknowledge a partial support from the Harvard College Observatory, and the Society of Fellows, Harvard University.

\section{References}

1. Wells, D. 1980, “Nonlinear Image Restoration: What We Have Learned”, Proc. S. P. I. E. 264, 148.

2. Wells, D. 1983, “Nonlinear Image Restoration", in the Proceedings of a Joint Topical Meeting on Information Processing in Astronomy and Optics, AAS/OSA publication, p. ThA10-1; and references therein.

3. Malin, D., and Carter, D. 1983, "A Catalog of Elliptical Galaxies with Shells", Astrophys. J. 274, 534; and references therein.

4. Schweizer, F., and Ford, K. 1985, "Fine Structure in Elliptical Galaxies", in J.-L. Nieto (ed.), Proceedings of the special IAU Colloquium "New Aspects of Galaxy Photometry", Springer Verlag Lectures in Physics Series 232, p. 145.

5. Schweizer, F. 1986, "Colliding and Merging Galaxies", Science 231, 227; and references therein.

6. Djorgovski, S., and Spinrad, H. 1985, “Surface Photometry of Comet P/Encke”, Astron. J. 90, 869.

7. Sekanina, Z., and Larson, S. 1984, “Coma Morphology and Dust-Emission Pattern of Periodic Comet Halley. I.", Astron. J. 89, 571 .

8. Sekanina, Z., and Larson, S. 1984, “Coma Morphology and Dust-Emission Pattern of Periodic Comet Halley. II.", Astron. J. 89, 1408.

9. Djorgovski, S. 1985, "Surface Photometry of Early-Type Galaxies", Ph. D. Thesis, U. C. Berkeley.

10. Lauer, T. R. 1985, “Boxy Isophotes, Discs and Dust Lanes in Elliptical Galaxies", M.N.R.A.S. $216,429$.

11. Djorgovski, S., and Ebneter, K. 1986, “A Search for Features in Early-Type Galaxies”, in J. Hearnshaw and P. Cottrell (eds.), Proceedings of the IAU Symposium \#118, D. Reidel, in press; and in preparation.

12. Sparks, W., et al. 1985, "Dust in Elliptical Galaxies", M.N.R.A.S. 217, 87.

13. Sadler, E., and Gerhard, O. “How Common are Dust Lanes in Early-Type Galaxies?”, M.N.R.A.S. 214, 177.

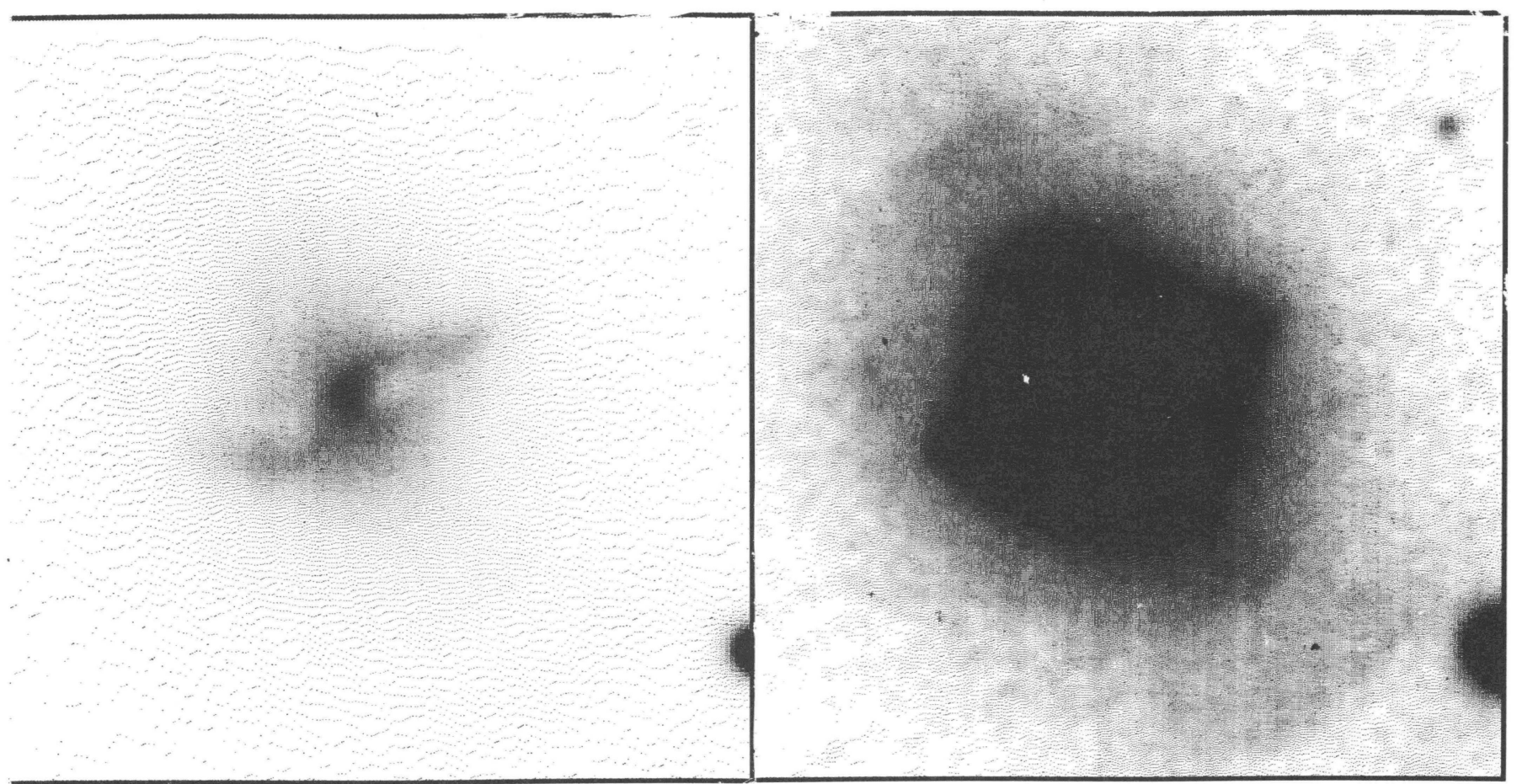

Figure 1. Low-contrast image of Arp 230 (Epoch 1950 coordinates $\left.\mathrm{RA}=00^{h} 43^{m} 56^{s}, \mathrm{DEC}=-13^{\circ} 43^{\prime} 10^{\prime \prime}\right)$. A sharp nucleus, warped stellar disk (ring?), and indications of a dust lane are evident.
Figure 2. The same image of Arp 230 as in Fig. 1, but displayed at 15 times the contrast. Note the appearance of an elliptical envelope perpendicular to the nuclear structure, and characteristic Malin-Carter shells. 


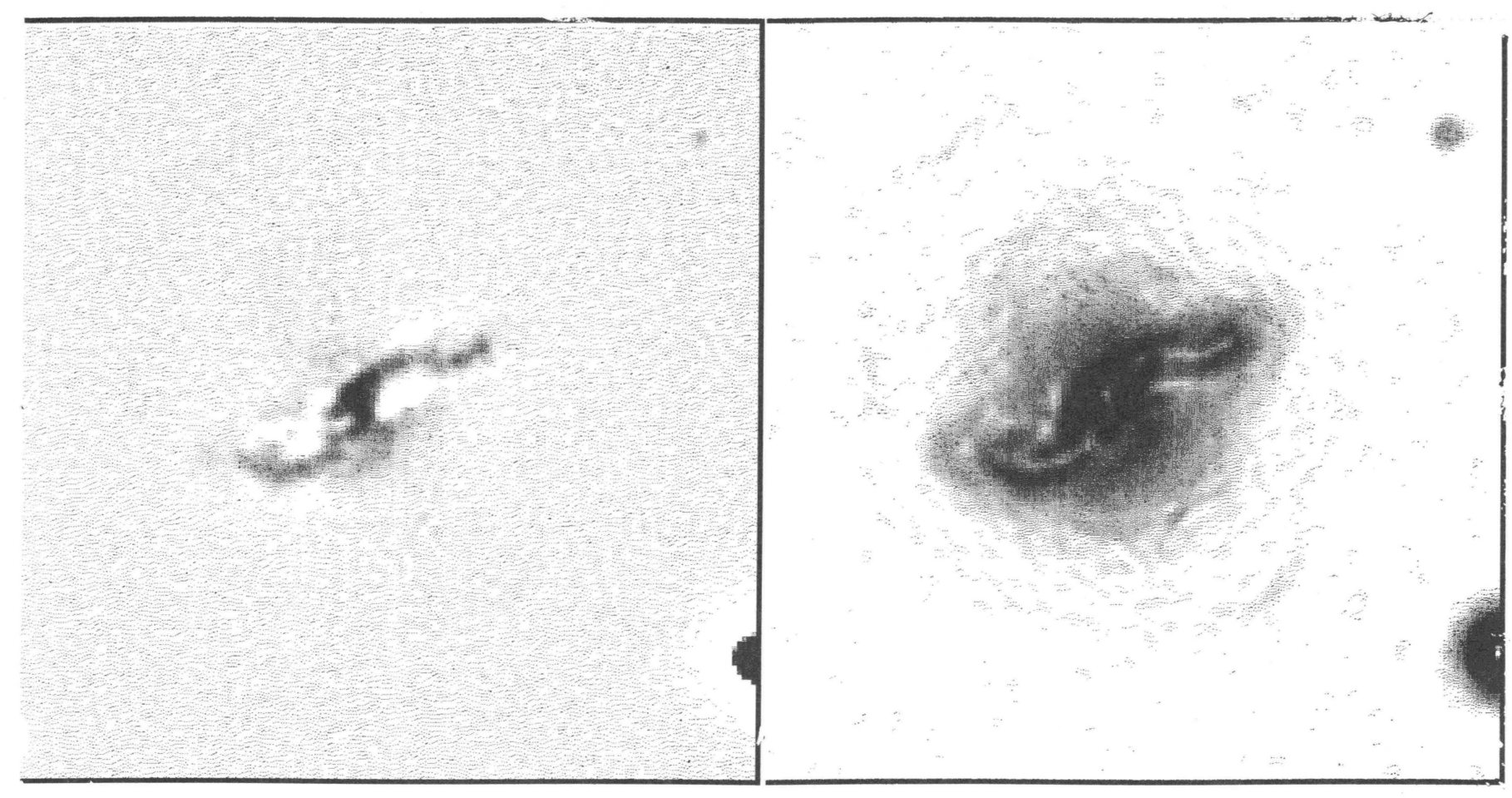

Figure 3. A product of high-pass Fourier filtering. Gibbs ringing near intensity peaks is very prominent, in the spite of a tapered filter.
Figure 4. The effect of a two-dimensional, Laplacean gradient filter (see the text, Eqs. 1 and 2). The shells are very weakly detected.

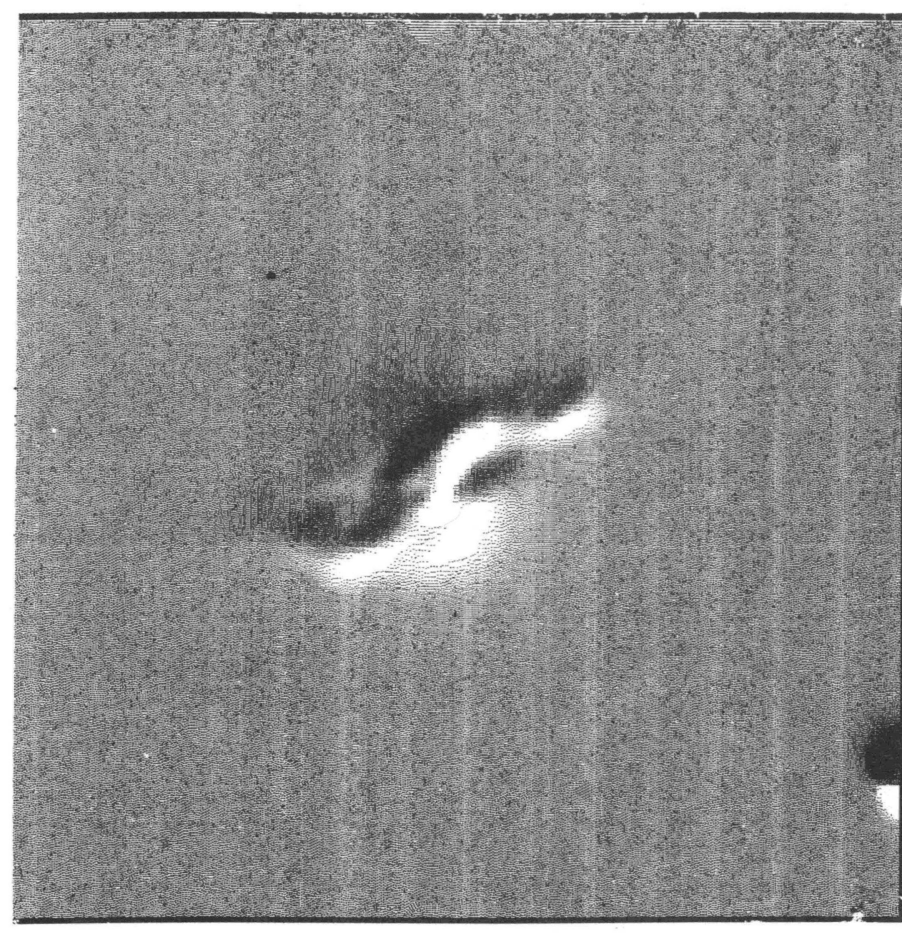

Figure 5. The effect of a directional gradient (subtraction version). The image was moved 1 pixel in $\mathrm{X}$ and 1.6 pixels in $\mathrm{Y}\left(\mathrm{PA} \sim 60^{\circ}\right)$, smoothed, and subtracted from the original.

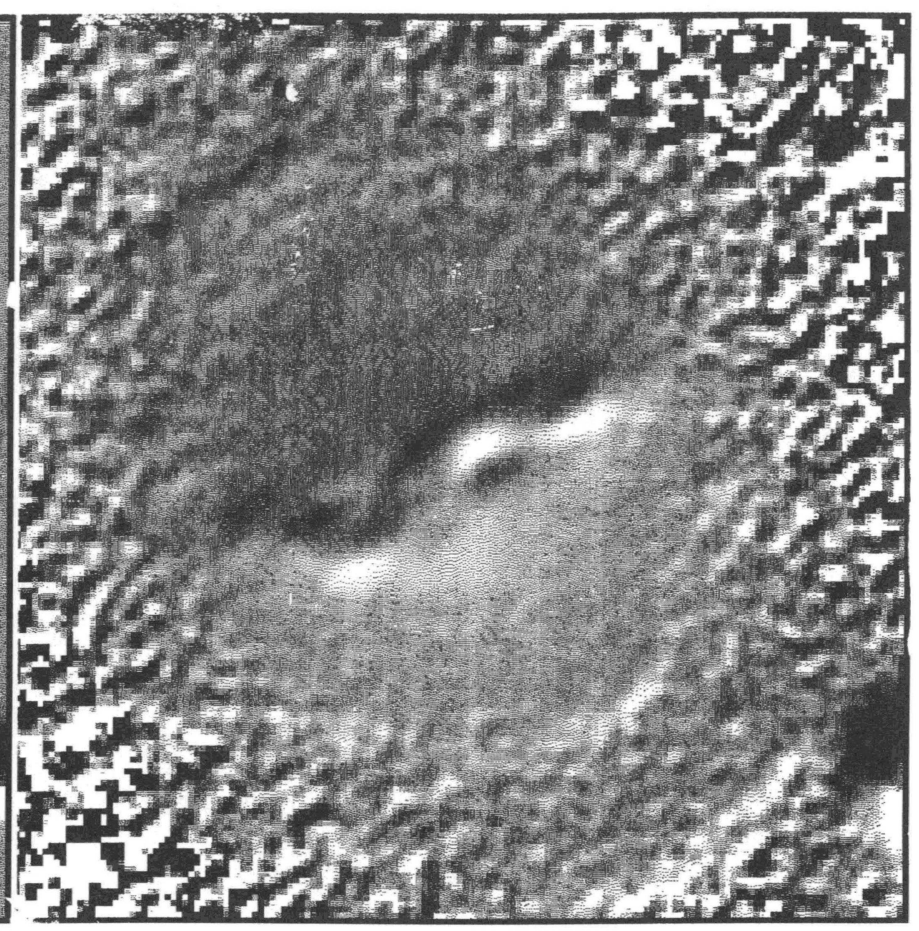

Figure 6. The effect of a directional gradient (division version). The faint outter structures (shells) become apparent, but the noise increases as well. 


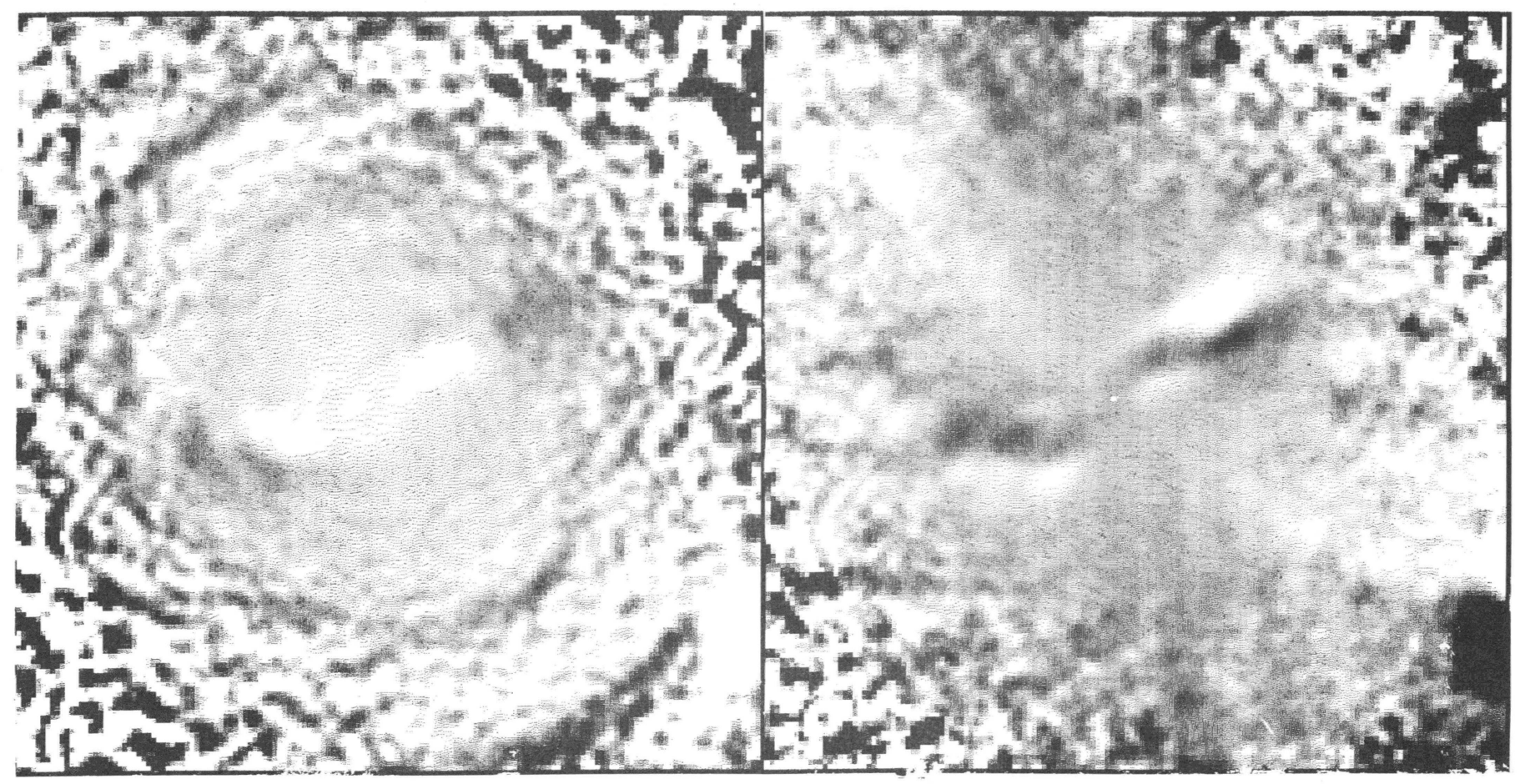

Figure 7. Polar coordinates gradient: radial stretch, but no rotation. The image was expanded radially by a factor of 1.05 , and divided by the smoothed original. The shells are very prominent.

Figure 8. Polar coordinates gradient: rotation, but no radial stretch. The image was rotated by $8^{\circ}$, and divided by the smoothed original. The shells are not visible, but the nuclear structure is highly enhanced.

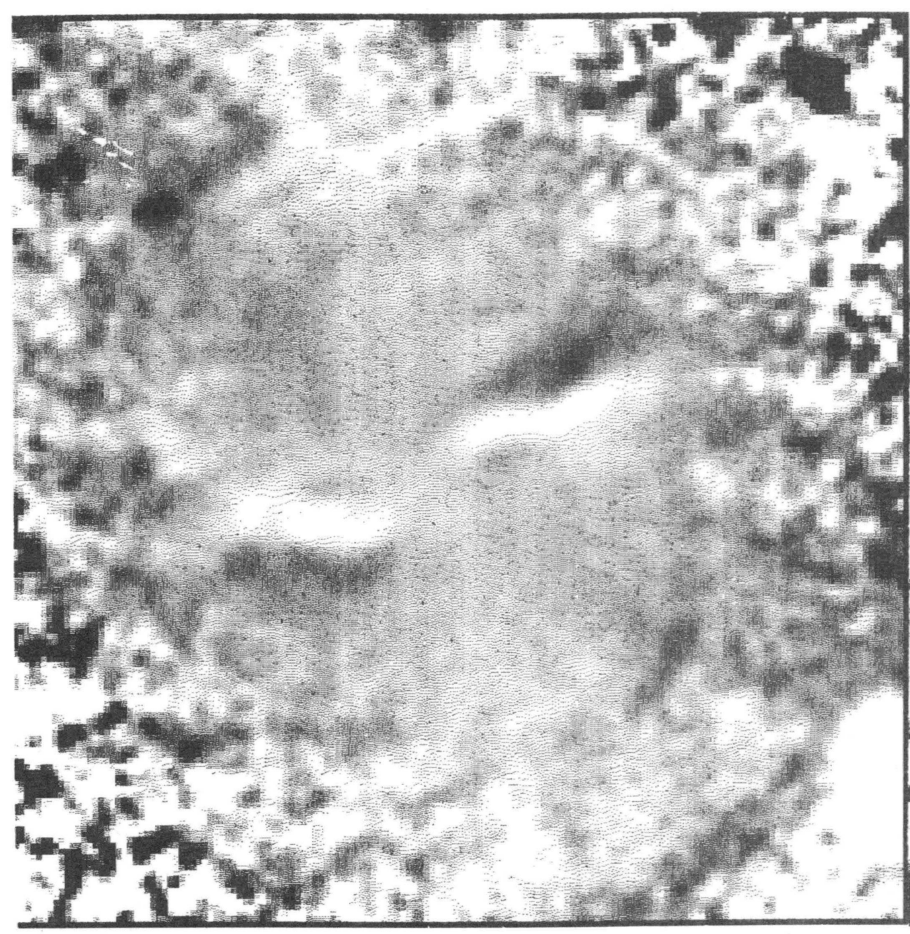

Figure 9. Polar coordinates gradient: rotation and radial stretch. The image was rotated by $7^{\circ}$, expanded radially by a factor of 1.05 , and divided by the smoothed original. All features are enhanced now.

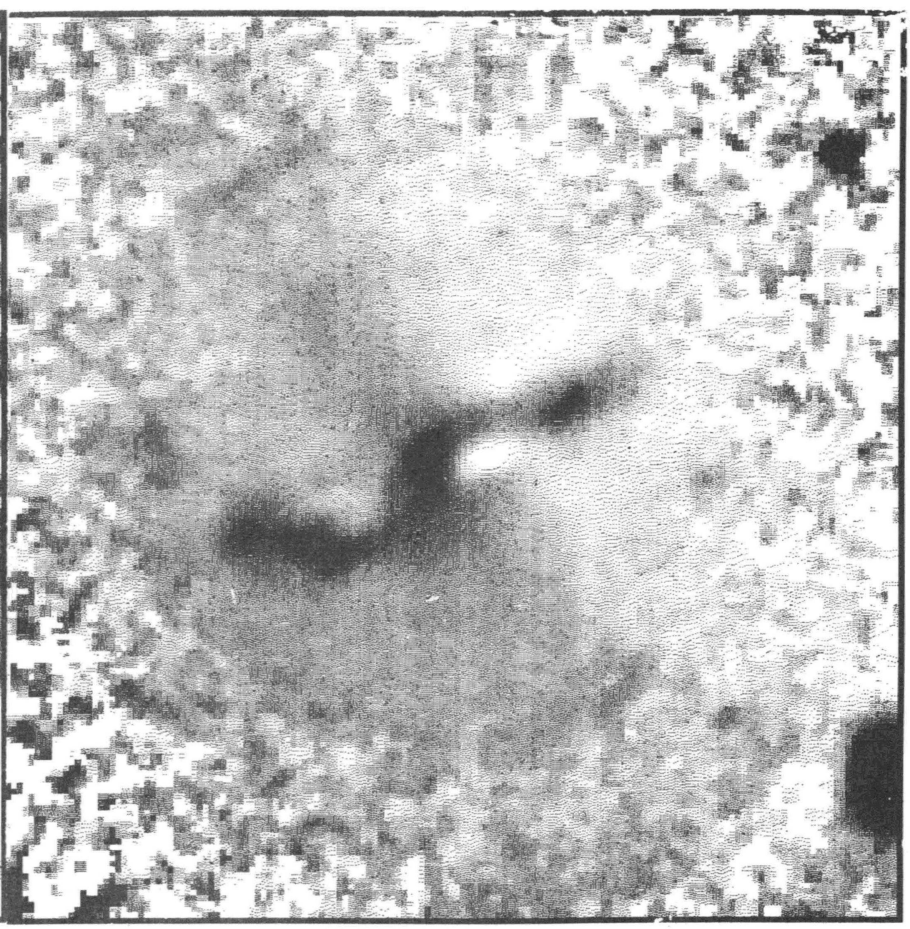

Figure 10. The effect of division by a smoothed (background) image. All features are enhanced. Note also the knotty character of the nuclear stellar disk/ring. 


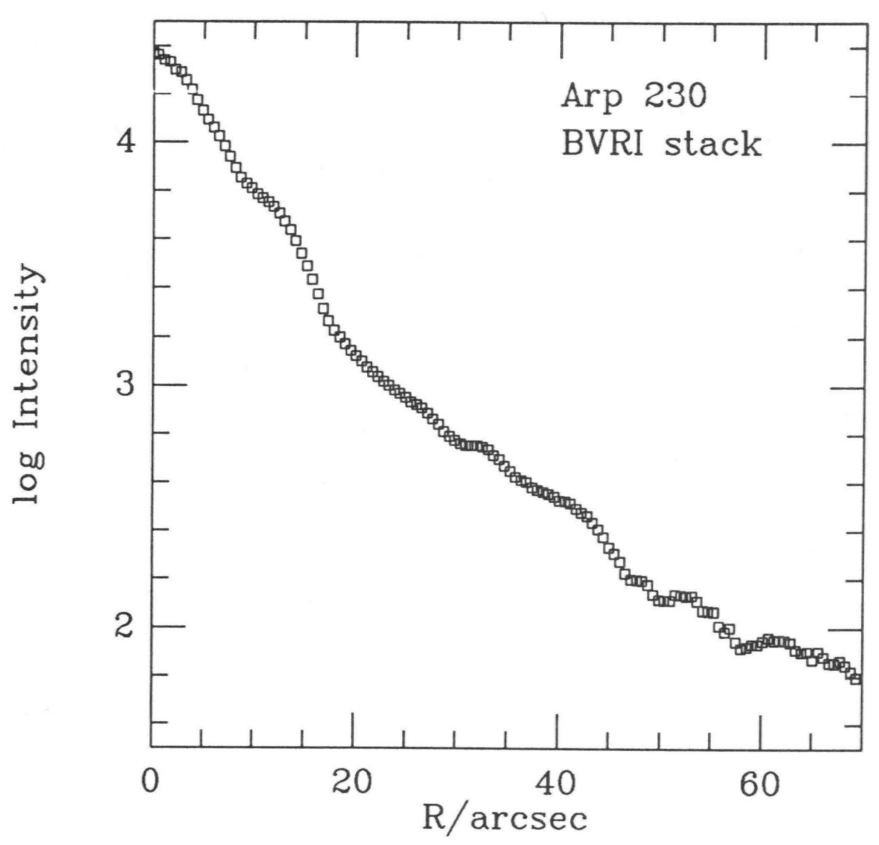

Figure 11a. Surface brightness (major axis) profile of Arp 230. The envelope is practically exponential (e-folding length $\sim 15$ arcsec), suggesting, perhaps, that both progenitors of Arp 230 were disk galaxies.

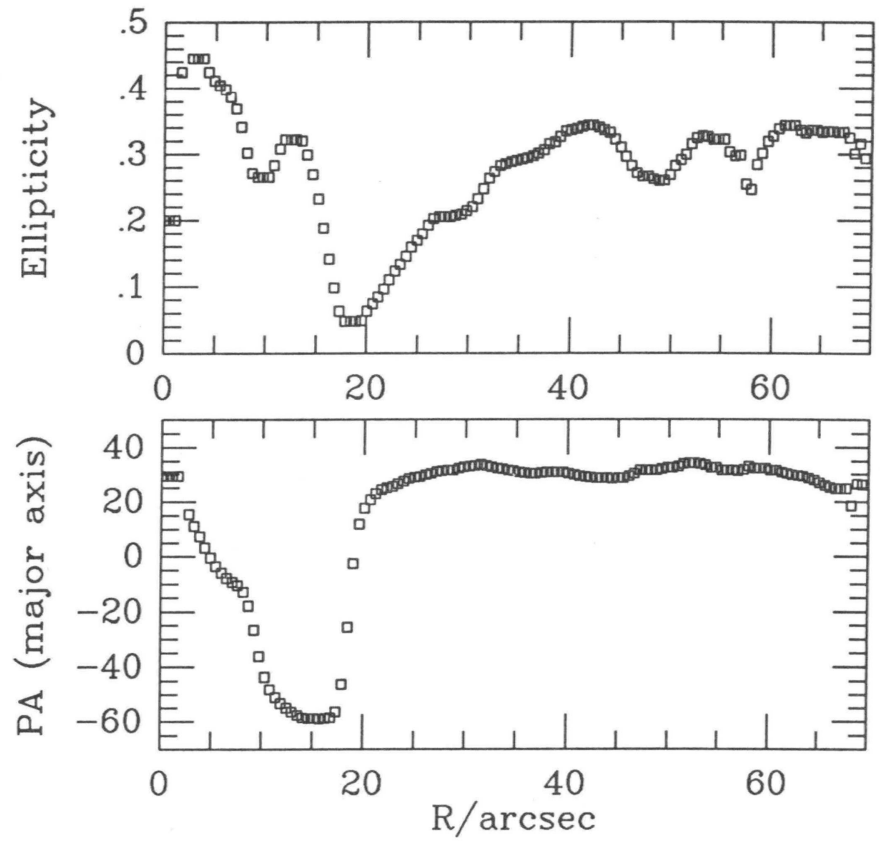

Figure 11b. Ellipticity and position angle profiles of Arp 230. The nuclear turmoil is clearly reflected in these profiles, but they stabilize beyond $\sim 20$ arcsec. The ellipticity dips at $\sim 48$ and $\sim 58$ arcsec may be a signature of the shells.

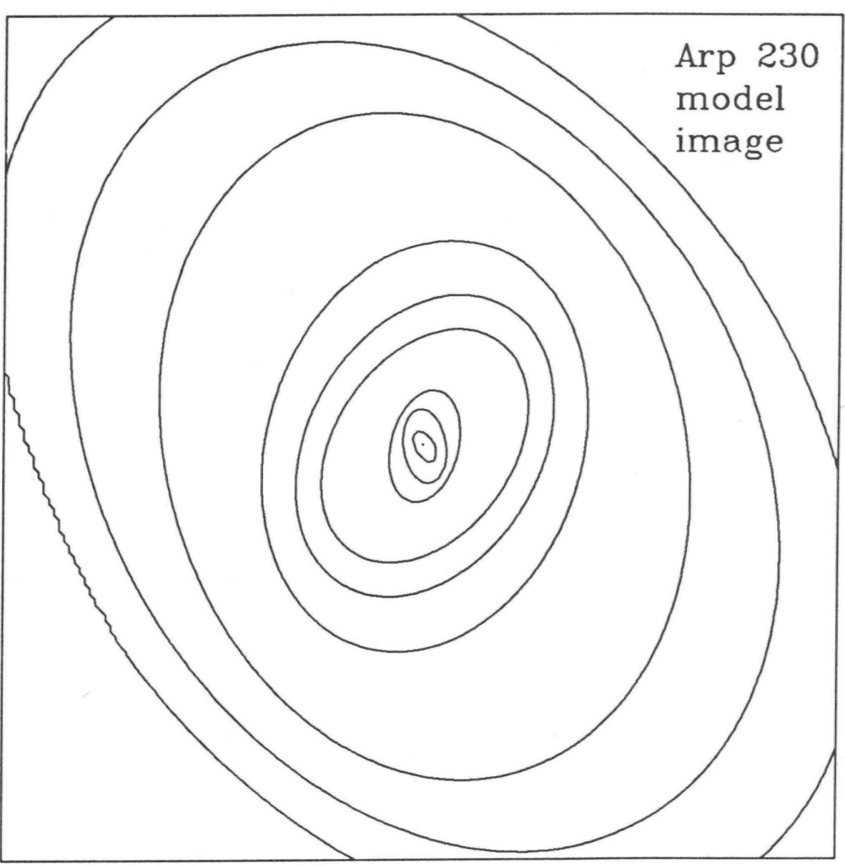

Figure 12. Contour map of the "reconstructed" image (elliptical isophote model) of Arp 230. The contour levels are at $0.5,1,2,5,10,20,50,70$, and 90 percent of the peak intensity.

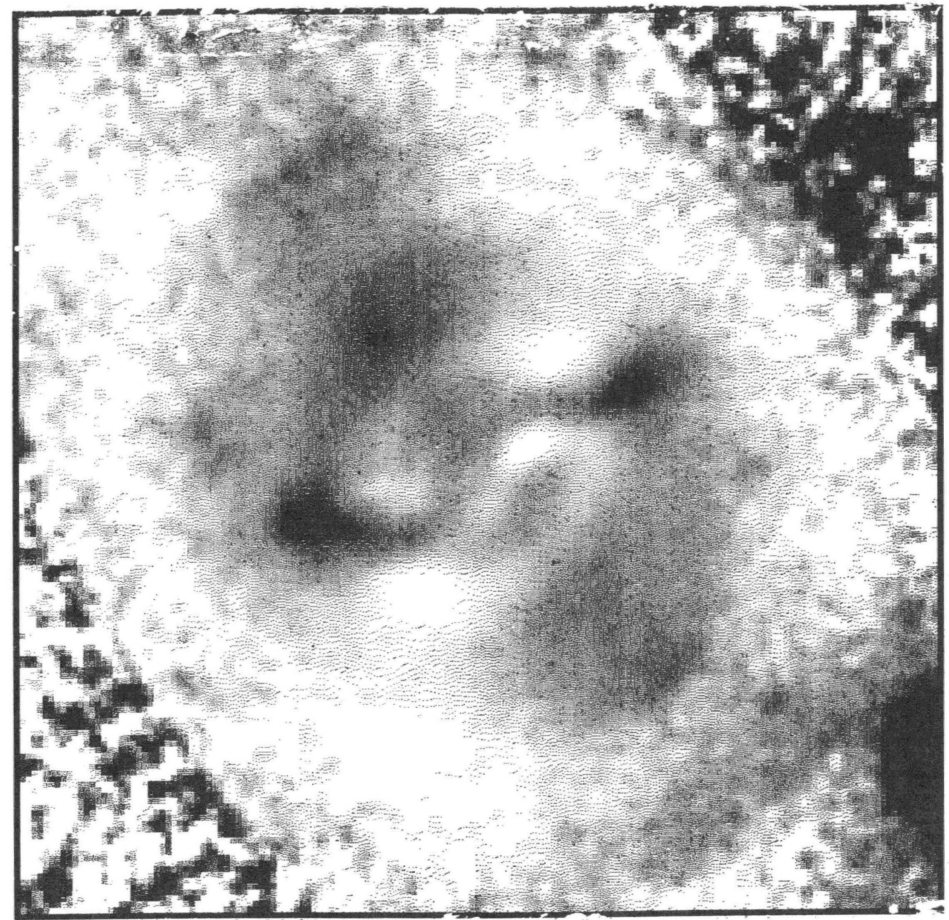

Figure 13. The original image divided by the elliptical model image. This is the best enhancement result so far, in the spite of a non-elliptical character of the inner isophotes of this galaxy. 